\title{
On Translating One Polyomino To Tile the Plane
}

\author{
D. Beauquier ${ }^{1}$ and $M$. Nivat ${ }^{2}$ \\ ${ }^{1}$ L.I.T.P., Université Paris VI, 2 Place Jussieu, \\ 75251 Paris Cédex 05, France \\ ${ }^{2}$ L.I.T.P., Université Paris VII, 2 Place Jussieu, \\ 75251 Paris Cédex 05, France \\ nivat (a) litp.ibp.fr
}

Communicated by Franco P. Preparata

\begin{abstract}
Given a polyomino, we prove that we can decide whether translated copies of the polyomino can tile the plane. Copies that are rotated, for example, are not allowed in the tilings we consider. If such a tiling exists the polyomino is called an exact polyomino. Further, every such tiling of the plane by translated copies of the polyomino is half-periodic. Moreover, all the possible surroundings of an exact polyomino are described in a simple way.
\end{abstract}

\section{Introduction}

The problem of tiling the plane has both theoretic and practical implications. From a theoretic viewpoint, it was proved by Wang [6] that the problem gives an alternative model of computation which has the same power as Turing machines, Post rewriting systems, and Markov algorithms. In practice, the problem appears in connection with the organization of data, given as an array of numbers or symbols where we want to optimize processing by means of a parallel computer [5], [7].

In [7] Wijshoff and Van Leeuwen prove the decidability of tiling the plane with one polyomino. In this paper we give a stronger result, with a simpler proof: a polyomino is exact, i.e., can tile the plane if and only if it can be completely surrounded by translated copies of itself (this condition is quite simple, and less strong than the one exhibited in [7]). Moreover, by a careful study of the properties of the word which describes the contour of the polyomino, we can prove that 
every tiling is half-periodic. This requires some technical lemmas, using essentially results of combinatorics on words. As a corollary of the proof of the halfperiodicity property, we obtain a complete description of the possible surroundings of an exact polyomino. Only three types of complete surroundings exist with, respectively, six, seven, or eight polyominoes and we can describe exactly how they are done.

\section{Preliminaries}

Let $E$ be a Euclidean plane and let $(Q, i, j)$ be an orthonormal reference system. A cell is a closed square in $E$, of unit area, whose vertices have integer coordinates. A polyomino is a finite union of nonoverlapping cells, whose outline is a single simple closed curve. So, a polyomino has no hole and its interior is a connected set. Two polyominoes are equivalent if there exists a translation of the plane which maps one onto the other. The equivalence class of a polyomino $p$ is a polyomino type $P$. Every polyomino type $P$ has a particular element, called its canonical element, namely the polyomino whose leftmost vertex among the highest ones is in the origin of the plane. Henceforth, a polyomino type $P$ is identified with its canonical element and with any of its elements if no ambiguity arises.

\subsection{Adjacency, Neighboring}

We give here general definitions which concern polyominoes of different types. Two polyominoes $p$ and $q$ (not necessarily of the same type) are called:

neighboring if they have a nonempty intersection, with empty interior; simply neighboring if they are neighboring and their intersection is a connected set;

adjacent if $p$ and $q$ are simply neighboring and their intersection is not reduced to a point.

The following diagram illustrates these definitions:

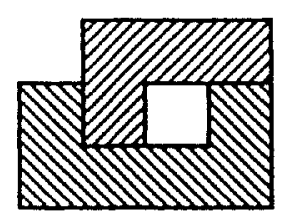

neighbouring but not simply neighbouring polyominoes

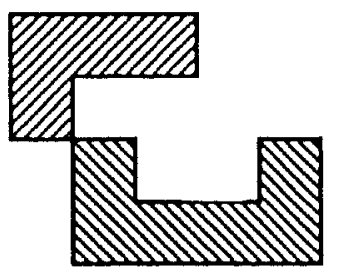

simply neighbouring but not adjacent polyominoes

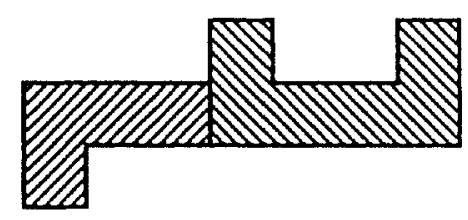

adjacent polyominoes 


\subsection{Boundary-Edges Boundary Between Two Simply Neighboring Polyominoes}

Note. Actually, the following definitions concern polyomino types, because these definitions are invariant under any translation of the polyominoes.

The boundary $b(p)$ of a polyomino $p$ is clockwise oriented. The length of the boundary is denoted by $|b(p)|$.

Let $X$ be the alphabet $\{l, r, u, d\}$ (for left, right, up, and down). With every point of $h(p)$ with integer coordinates, there is associated a word $m$ on the alphabet $X$, which describes the sequence of moves along the boundary, starting from an origin $A$. When the origin is changed, the word obtained is a conjugate of $m$. We can observe that $|m|=|b(p)|$. The word $m$ is called the label of the boundary $h(p)$ with

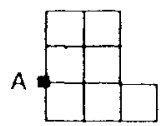

the origin in $A$. The label of $h(p)$ with origin $A$ is "uurrddrdlllu" in the example given above.

Let $A$ and $B$ be two points of $b(p)$ with integer coordinates. The edge $[A B]$ is the path from $A$ to $B$ along the boundary $b(p)$. The label of this edge is defined in the same way as the label of $h(p)$, and is written $\langle A B\rangle$.

Remark. $\langle A A\rangle=1$. If $B \in[A C]$, then $\langle A B\rangle\langle B C\rangle=\langle A C\rangle$.

Let $p$ be a polyomino and let $u$ be a vector of $Z^{2}$. We denote by $p_{u}$ the image of $p$ under the translation of vector $u$. Let $p_{u}$ and $q_{r}$ be two simply neighboring polyominoes, and $A$ and $B$ are the endpoints of their intersection such that the intersection edge goes from $A$ to $B$ on $b\left(p_{u}\right)$ and from $B$ to $A$ on $b\left(q_{1}\right)$. Let $A_{1}$ and $B_{1}$ (resp. $A_{2}$ and $B_{2}$ ) be the images of $A$ and $B$ in the translation of vector $-u$ (resp. $-v$ ). The edge $\left[A_{1} B_{1}\right]$ is called the boundary of $p_{u}$ with respect to $q_{v}$ and is denoted by $\left[p_{u}, q_{v}\right]$. The edge $\left[B_{2} A_{2}\right]$ is the boundary of $q_{v}$ with respect to $p_{u}$ and is written $\left[q_{v}, p_{u}\right]$. The label of $\left[p_{u}, q_{1}\right]$ is denoted by $\left\langle p_{u}, q_{v}\right\rangle$. We agree to represent in $p_{u}$ and $q_{r}$ the points $A_{1}, B_{1}, A_{2}, B_{2}$ as is done below:

$A_{2}$ is in the origin of the plane. We draw the diagram like this:
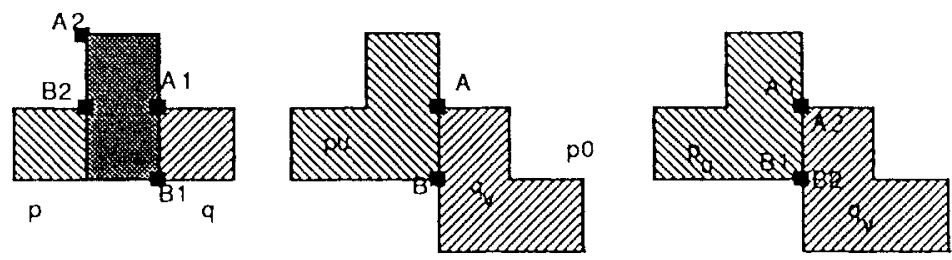

$$
\begin{aligned}
& {\left[p_{u}, q_{v}\right]=\left[A_{1} B_{1}\right] \text { and }\left\langle p_{u}, q_{v}\right\rangle=\left\langle A_{1} B_{1}\right\rangle=d d,} \\
& {\left[q_{v}, p_{u}\right]=\left[B_{2} A_{2}\right] \text { and }\left\langle q_{v}, p_{u}\right\rangle=\left\langle B_{2} A_{2}\right\rangle=u u}
\end{aligned}
$$


Let $T$ be the involution of $X^{*}$ recursively defined as follows:

$$
T(u)=d, \quad T(d)=u, \quad T(r)=l, \quad T(l)=r,
$$

and

$$
\forall u \in X^{*}, \quad \forall x \in X, \quad T(u x)=T(x) T(u)
$$

$T(u)$ will be denoted by $u^{2}$.

Remark. $\langle p, q\rangle^{\sim}=\langle q, q\rangle$.

We give here some basic results established in [7]:

Lemma 2.1. Let $q$ be a polyomino such that $q$ and $q_{u}$ do not overlap. Then $q, q_{u}$, $q_{2 u}$ are pairwise nonoverlapping.

Lemma 2.2. Let $q, q_{u}, q_{v}$ be three polyominoes two by two neighboring. Then $q$, $q_{u}, q_{v}, q_{u-v}, q_{-u}, q_{-v}, q_{t-u}$ are pairwise nonoverlapping.

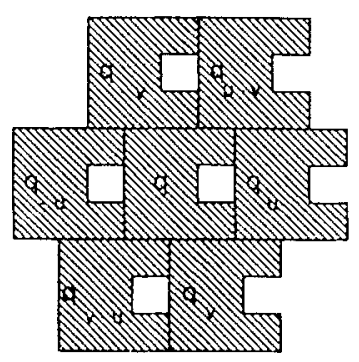

Lemma 2.3. Given a polyomino $q$ with boundary $B$, let us consider two adjacent polyominoes $q$ and $q_{u}$ forming a hole. Then the size of the interior boundary $I$ of the union of these polyominoes with respect to $h$ is strictly less than $|B|$.

\subsection{Surroundings}

Let $p$ be a polyomino. A surrounding of $p$ is a circular sequence $\left(p_{0}, \ldots, p_{k-1}\right)$ of polyominoes (circular means that the sequence is defined up to a circular permutation), such that, for $i=0, \ldots, k-1, p$ and $p_{i}$ are simply neighboring, as also are $p_{i}$ and $p_{i+1}$ (indices are defined modulo $k$ ), and, moreover, $A_{i+1}=B_{i}$ (where $\left[p, p_{i}\right]=\left[A_{i} B_{i}\right]$ ).

The surrounding is

complete if, for every $i, p_{i}$ and $p_{i+1}$ are adjacent, and minimal if, for every $i,\left|\left[p, p_{i}\right]\right| \neq 0$. 


\section{Examples.}

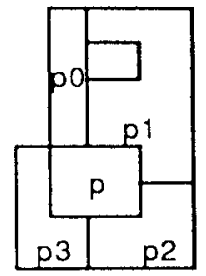

(a)

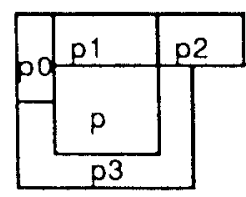

(b)

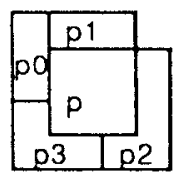

(c)

In case (a), $\left(p_{0}, p_{1}, p_{2}, p_{3}\right)$ is not a surrounding of $p$ because $p_{0}$ and $p_{1}$ are not simply neighboring. In case (b), $\left(p_{0}, p_{1}, p_{2}, p_{3}, p_{4}\right)$ is a complete surrounding of $p$. In case $(\mathrm{c}),\left(p_{0}, p_{1}, p_{2}, p_{3}\right)$ is a minimal surrounding of $p$.

We can observe that the concatenation of edges $\left[A_{i} A_{i+1}\right]$ for $i=0, \ldots, k-1$ is exactly the boundary $b(p)$ with the origin in $A_{0}$, and the concatenation of the labels $\left\langle A_{i} A_{i+1}\right\rangle$ is the label of this boundary.

Notation. If $A$ is a point on the boundary of $p$ with integer coordinates, we denote its opposite point on this boundary by $A^{\prime}$, that is the point $A^{\prime}$ on $b(p)$ such that $\left|\left[A A^{\prime}\right]\right|=\left|\left[A^{\prime} A\right]\right|$.

\section{Tilings}

Let $P$ be a polyomino type and let $p$ be its canonical element. A tiling $T$ of the plane $E$ by $P$ is defined by a subset $T$ of $Z^{2}$ such that $E$ is the union of the polyominoes $p_{u}$ for $u$ in $T$, and, for two different vectors $u$ and $v$ of $T, p_{u}$ and $p_{v}$ are disjoint or neighboring. We observe that if, in a tiling $T$, two polyominoes $p_{u}$ and $p_{v}$ are neighboring, then they are simply neighboring, because of Lemma 2.3.

A polyomino is said to be exact if there exists a tiling of the plane by this polyomino.

A tiling $T$ is periodic if there exists two linearly independent vectors $u$ and $v$ of $Z^{2}$ such that $T$ is not changed by the corresponding translations. A tiling $T$ is regular if there are three vectors $u_{0}, u, v$ such that

$$
T=u_{0}+\left\{k u+k^{\prime} v /\left(k, k^{\prime}\right) \in Z^{2}\right\}
$$

A tiling $T$ is half-periodic if there is a vector $u \neq 0$ in $Z^{2}$ such that $T$ is unchanged under the translation of vector $u$.

Observe that every regular tiling is periodic and that a periodic tiling is half-periodic. 


\section{Examples.}

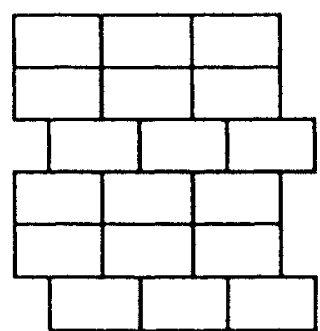

A nonregular but periodic tiling

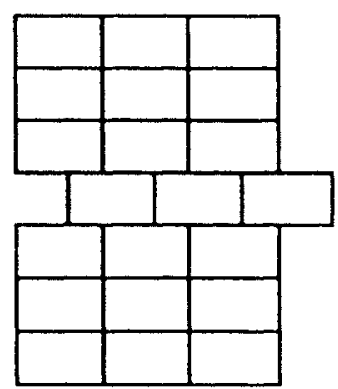

A half-period but nonperiodic tiling

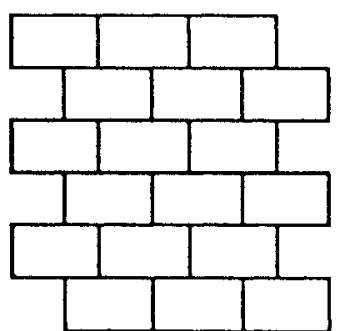

A regular tiling

Our purpose is to prove the following results:

1. Given a polyomino $p$, we can decide whether $p$ is exact.

2. If $p$ is exact:

(a) There exist regular tilings of $E$ by $p$.

(b) Every tiling of $E$ by $p$ is a half-periodic tiling.

A triad is a triple $(p, q, r)$ of polyoninoes of the same type which two by two are simply neighboring and such that $[p, q]$ is followed by $[p, r]$ in the boundary of $p$ and, moreover, the union of $p, q$, and $r$ is without hole. It induces the existence of a unique point common to $p, q$, and $r$.

If we have $[p, q]=\left[A_{1} A\right],[q, r]=\left[B_{1} B\right],[r, p]=\left[C_{1} C\right]$, then $(A, B, C)$ is called the contact of the triad $(p, q, r)$.

\section{Examples.}

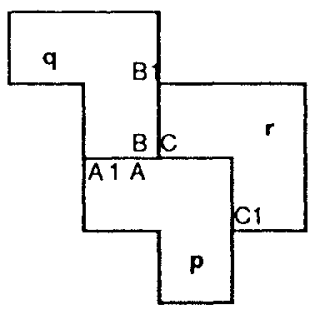

(a)

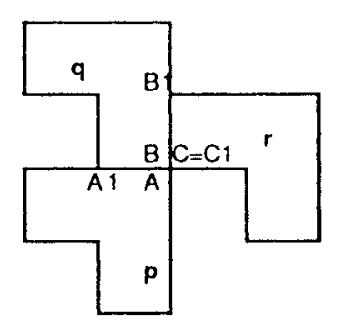

(b)

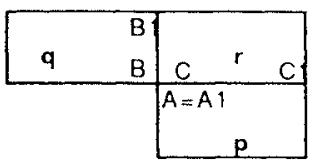

(c)

A contact is said to be exact if there is at least one tiling of the plane by the corresponding polyomino type $P$ in which the contact appears.

Remark. (a) and (c) are exact contacts, but not (b).

Theorem 3.1. Let $p$ be a polyomino. We can decide whether $p$ is exact. 
Proof. Since a tiling of the plane by a polyomino $p$ cannot contain two polyominoes which are neighboring but not simply neighbouring, clearly, if $p$ is exact, then it admits a surrounding with other polyominoes of the same type. So, Theorem 3.1 is a consequence of the following proposition.

Proposition 3.1. Let $p$ be a polyomino which admits a surrounding. Then $p$ is an exact polyomino and, moreover, if $p$ is exact there exists a regular tiling of the plane by this polyomino.

Proof. Let $u_{0}, \ldots, u_{k-1}$ be $k$ vectors of $\mathbf{Z}^{2}$ such that $\left(p_{u_{0}}, \ldots, p_{u_{k-1}}\right)$ is a surrounding of $p$. Let us define the following boundaries, more exactly their related labels:

$$
b_{i}=\left\langle p, p_{u_{i}}\right\rangle \quad \text { and } \quad b_{i}^{\prime}=\left\langle p_{u_{t}}, p_{u_{i+1}}\right\rangle \quad \text { for } \quad i=0, \ldots, k-1
$$

(Index integers are defined modulo $k$.) Using translation we have

$$
\begin{aligned}
\left\langle p, p_{u_{i}}\right\rangle & =\left\langle p_{u_{i}}, p_{2 u_{i}}\right\rangle=\left\langle p_{u_{i+1}}, p_{u_{i}+u_{i+1}}\right\rangle=b_{i} \\
\left\langle p, p_{u_{i+1}}\right\rangle & =\left\langle p_{u_{i}}, p_{u_{i}+u_{i+1}}\right\rangle=\left\langle p_{u_{i+1}}, p_{2 u_{i+1}}\right\rangle=b_{i+1}, \\
\left\langle p_{u_{i}}, p_{u_{i+1}}\right\rangle & =\left\langle p_{2 u_{i}}, p_{u_{i}+u_{i+1}}\right\rangle=\left\langle p_{u_{i}+u_{i+1}}, p_{2 u_{i+1}}\right\rangle=b_{i}^{\prime}
\end{aligned}
$$

Consequently, the triads $\left(p, p_{u_{1}}, p_{u_{i}, 1}\right),\left(p_{u_{1}}, p_{2 u_{1}}, p_{u_{i}+u_{i}, 1}\right),\left(p_{u_{i+1}}, p_{u_{i}+u_{i}, 1}, p_{2 u_{i+1}}\right)$ are the same.

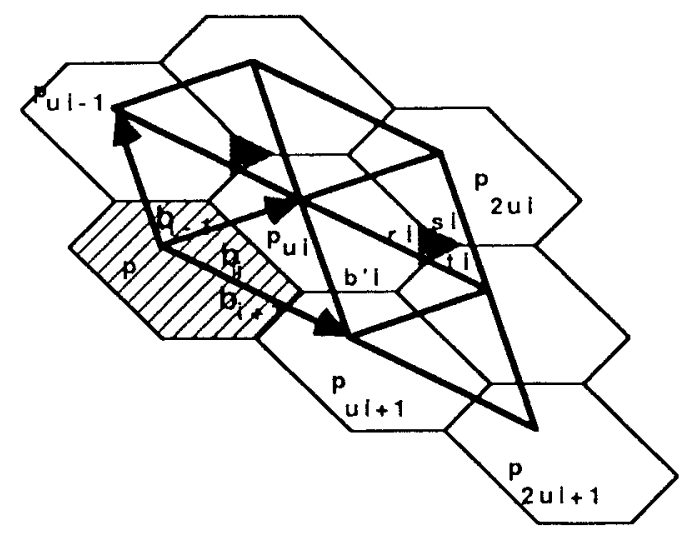

On the other hand, it is not certain that the triple $\left(p_{u_{i+1}}, p_{u_{i}}, p_{u_{i}+u_{i+1}}\right)$ is a triad. Actually, the polyominoes, of course, are two by two simply neighboring but perhaps the union has a hole.

Let $r_{i}$ (resp. $s_{i}, t_{i}$ ) be the label of the orientated edge of the boundary of $p_{u_{i}}$ (resp. $\left.p_{u_{i}+u_{t+1}}, p_{u_{t+1}}\right)$ whose origin is the end of $\left[p_{u_{i}}, p_{u_{i}+u_{i+1}}\right]$ (resp. $\left[p_{u_{i}}, p_{u_{t+1}}\right],\left[p_{u_{i}+1}, p_{u_{t}}\right]$ ) and whose end is the origin of $\left[p_{u_{t}}, p_{u_{t+1}}\right]$ (resp. $\left[p_{u_{1}+u_{t+1}}, p_{u_{l}}\right],\left[p_{u_{t+1}}, p_{u_{t}+u_{i+1}}\right]$ ) (see the diagram above).

We can observe that the concatenation $r_{i} s_{i} t_{i}$ is the label of the hole in the union 
$p_{u_{i+1}} \cup p_{u_{i}} \cup p_{u_{i}+u_{i+1}}$. Finally, if $\left|r_{i}\right|=0$, then since $p_{u_{i+1}}$ and $p_{u_{i}+u_{t+1}}$ are simply neighboring, it implies that $\left|s_{i}\right|=\left|t_{i}\right|=0$ (similarly, if $s_{i}$ or $t_{i}$ is the empty word, then the other two are empty). Now, if we compute the length of the boundary of $p$, by looking at the polyomino $p_{u_{i}}\left(\right.$ resp. $p_{u_{1}+u_{i+1}}$ ) and if we sum for $i=0$ up to $k-1$, comparing the two results we conclude that the sum of the $\left|s_{i}\right|$ is equal to zero:

$$
|b(p)|=2\left|b_{i}\right|+\left|b_{i+1}\right|+\left|b_{i-1}\right|+\left|b_{i}^{\prime}\right|+\left|b_{i-1}^{\prime}\right|+\left|r_{i}\right|+\left|t_{i-1}\right|
$$

Let us $\operatorname{sum}$ for $i=0$ to $k-1$ :

$$
k|b(p)|=4 \sum\left|b_{i}\right|+2 \sum\left|b_{i}^{\prime}\right|+\sum\left|r_{i}\right|+\sum\left|t_{i}\right|
$$

Computing in the same way the length of the boundary of $p_{u_{1}+u_{1+1}}$, we have

$$
|b(p)|=2\left|b_{i}\right|+2\left|b_{i}^{\prime}\right|+2\left|b_{i+1}\right|+\left|s_{i}\right|+\left|t_{i}\right|+\left|r_{i}\right| \text {. }
$$

The sum is

$$
k|b(p)|=4 \sum\left|b_{i}\right|+2 \sum\left|b_{i}^{\prime}\right|+\sum\left|s_{i}\right|+\sum\left|t_{i}\right|+\sum\left|r_{i}\right|
$$

Comparing (1) and (2) we conclude $\left|s_{i}\right|=\left|r_{i}\right|=\left|t_{i}\right|=0$ for every $i$. We can conclude that $\left(p_{u_{i+1}}, p_{u_{i}}, p_{u_{i}+u_{t+1}}\right)$ is a triad for every $i$ and that $\left(p_{u_{i}-u_{i+1}}, p_{u_{i}}, p_{u_{i+1}}, p_{u_{i+1}-u_{i}}, p_{-u_{i}}\right.$, $p_{-u_{1+1}}$ ) is a surrounding of $p$ (look at the surrounding of $p_{u_{1}+u_{1},}$, below).

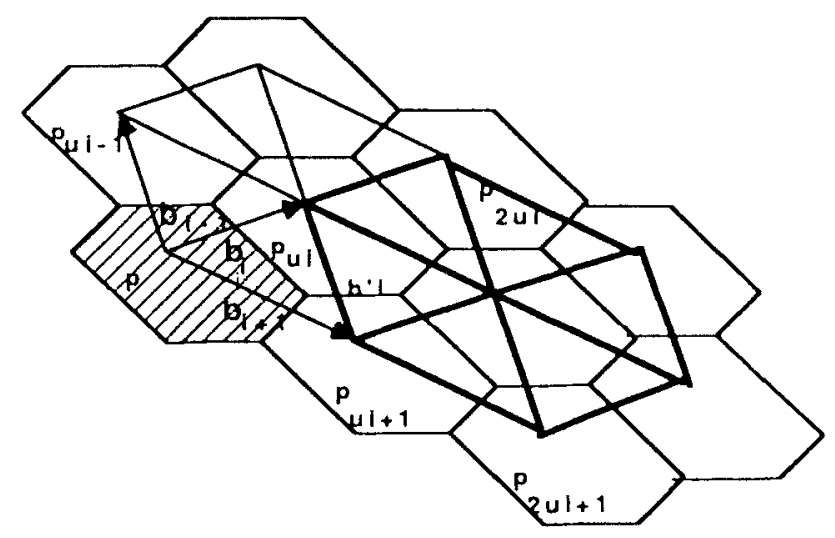

This surrounding can be translated whence the set $T=\left\{k u_{i}+k^{\prime} u_{i+1} /\left(k, k^{\prime}\right) \in \mathbf{Z}^{2}\right\}$ is a regular tiling of the plane by $p$, and this holds for each $i$.

Corollary 3.1. Every surrounding of a polyomino can be extended to a tiling of the whole plane, and consequently every contact appearing in a surrounding is an exact one. 
It now remains just to prove that if a polyomino $p$ is exact, then every tiling of the plane by $p$ is half-periodic. For that, we need two lemmas that follow directly from the proof of Proposition 3.1.

Lemma 3.1. Let $\mathbf{P}$ be a tiling of the plane with a polyomino type $P$. Let $(p, q, r)$ be a triad in this tiling whose contact is $(A, B, C)$. Then we have

$$
\begin{array}{lll}
{[p, q]=\left[B^{\prime} A\right],} & {[q, r]=\left[C^{\prime} B\right],} & {[r, p]=\left[A^{\prime} C\right],} \\
{[q, p]=\left[B A^{\prime}\right],} & {[r, q]=\left[C B^{\prime}\right],} & {[p, r]=\left[A C^{\prime}\right] .}
\end{array}
$$

Proof. If $(p, q, r)$ is a triad of $P$, then $q$ and $r$ are two consecutive polyominoes of a surrounding of $p$ (not necessarly complete) in $P$. So, $p, q$, and $r$ are like $p$, $p_{u_{1}}$, and $p_{u_{i+1}}$ in the proof of Proposition 3.1, and since $r_{i}, s_{i}$, and $t_{i}$ are reduced to points, Lemma 3.1 holds.

Lemma 3.2 (under the same hypothesis). If $u$ and $v$ are two vectors such that $q=p_{u}$ and $r=p_{v}$, then $\left(p_{u}, p_{v}, p_{v-u}, p_{-u}, p_{-v}, p_{u-v}\right)$ is a surrounding of $p$ (see the diagram below).

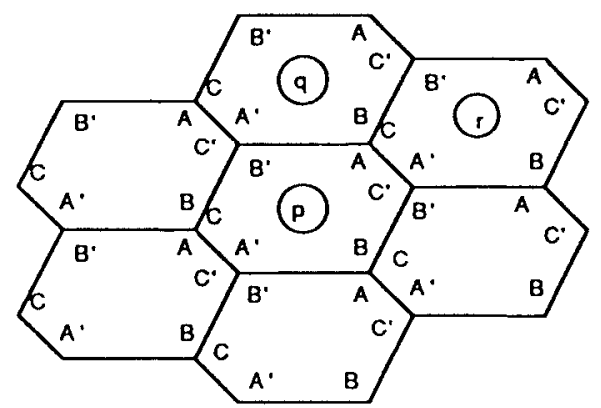

Proof. We consider $p, q, r$ as $p, p_{u_{i}}, p_{u_{1}+1}$ in the proof of Proposition 3.1. So we apply (3) and Lemma 3.2 is proved.

A polyomino $p$ is a pseudohexagon $A B C A^{\prime} B^{\prime} C^{\prime}$ if there exist three points $A, B$, $C$ on the boundary of $P$ such that $B \in[A C]$ and $\left\langle A^{\prime} B^{\prime}\right\rangle=\langle A B\rangle,\left\langle B^{\prime} C^{\prime}\right\rangle=\langle B C\rangle$, $\left\langle C^{\prime} A\right\rangle=\left\langle C A^{\prime}\right\rangle$. (It is equivalent to write that there exists a point $A$ such that the label of $b(P)$ starting at $A$ can be written $u v w u^{\sim} v^{\sim} w^{\sim}$.)

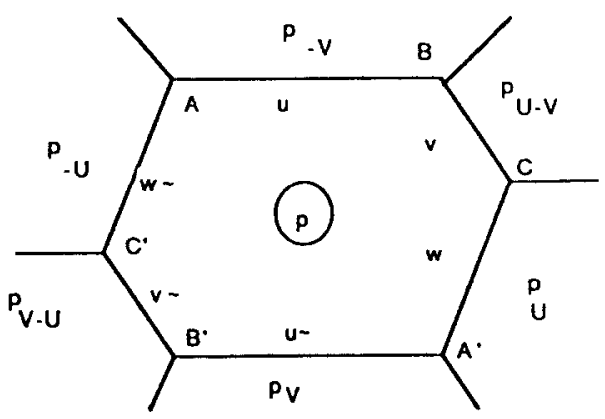


The previous results can now be stated in the following form:

Theorem 3.2. A polyomino $p$ is exact iff it is a pseudohexagon.

Proof. If $p$ is an exact polyomino, then Lemma 3.1 proves that $p$ is a pseudohexagon, considering that if $q$ and $r$ are two simply neighbor instances of $p$, then $\langle q, r\rangle=\langle r, q\rangle$. Now, if we write $\left\langle B^{\prime} A\right\rangle=u\left\langle A C^{\prime}\right\rangle=v$ and $\left\langle C^{\prime} B\right\rangle=w$, then $p$ is a pseudohexagon.

Conversely, let $U=A C$ and $V=B A^{\prime}$. Then $\left(p_{U}, p_{V}, p_{V-U}, p_{-U}, p_{-V}, p_{U-V}\right)$ is a surrounding of $p$ and so $p$ is an exact polyomino.

The following statement is a clear consequence of Lemma 3.2 and Theorem 3.2:

Corollary 3.2. If $(A, B, C)$ is an exact contact for the polyomino $p$, then $p$ is a pseudohexagon $B^{\prime} A C^{\prime} B A^{\prime} C$ and conversely.

\section{Half-Periodicity}

From now on, $p$ is an exact polyomino.

Lemma 4.1 is quite important for the proof of half-periodicity.

Lemma 4.1. If $\left(A, C, B^{\prime}\right)$ and $\left(A, D, B^{\prime}\right)$ are two different exact contacts $(C \neq D)$, such that $C \in[B D]$ and $B \neq C$ or $D \neq A^{\prime}$, then the following properties are satisfied: there exists a prime word $\alpha=\alpha_{1} \alpha_{2}$ and a conjugate $\alpha_{c}=\alpha_{2} \alpha_{1}$ of $\alpha$ such that

$$
\begin{aligned}
& \left\langle B A^{\prime}\right\rangle \in \alpha \alpha^{+}, \quad\left\langle B^{\prime} A\right\rangle \in \alpha_{\mathrm{c}}^{\alpha^{2} \alpha_{\mathrm{c}}^{+},} \\
& \langle C D\rangle \in \alpha_{c}^{+}, \quad\left\langle C^{\prime} D^{\prime}\right\rangle \in \alpha_{c}^{-+}, \\
& \langle B C\rangle \in \alpha^{*} \alpha_{1}, \quad\left\langle B^{\prime} C^{\prime}\right\rangle \in \alpha_{1}^{\tilde{\alpha}} \alpha^{\sim^{*}}=\alpha_{c}^{\alpha^{*}} \alpha_{1}^{\tilde{1}}, \\
& \left\langle D A^{\prime}\right\rangle \in \alpha_{2} \alpha^{*}, \quad\left\langle D^{\prime} A\right\rangle \in \alpha^{*} \alpha_{2}^{\tilde{2}}=\alpha_{2}^{\tilde{2}} \alpha_{\mathrm{c}}^{\tilde{u}^{*}} \text {. }
\end{aligned}
$$

Proof. Let $\langle B C\rangle=a,\langle C D\rangle=b$, and $\left\langle D A^{\prime}\right\rangle=c$. Then $\left\langle C A^{\prime}\right\rangle=b c$. We have $\left\langle C^{\prime} A\right\rangle=\left\langle C A^{\prime}\right\rangle^{\sim}=(b c)^{\sim}=c^{\sim} b^{\sim}$. On the other hand,

$$
\left\langle C^{\prime} A\right\rangle=\left\langle C^{\prime} D^{\prime}\right\rangle\left\langle D^{\prime} A\right\rangle=\left\langle C^{\prime} D^{\prime}\right\rangle\left\langle D A^{\prime}\right\rangle^{\sim}=\left\langle C^{\prime} D^{\prime}\right\rangle c^{\sim} .
$$

Letting $\left\langle C^{\prime} D^{\prime}\right\rangle=d \neq 1$ we have the equation

$$
c^{\sim} b^{\sim}=d c^{\sim},
$$

and this implies that there exists a primitive word $\alpha=\alpha_{1} \alpha_{2}$ and a conjugate of $\alpha$, $\alpha_{c}=\alpha_{2} \alpha_{1}$ such that

$$
d \in \alpha^{+}, \quad b^{\sim} \in \alpha_{c}^{\sim}, \quad c^{\sim} \in \alpha^{*} \alpha_{1} .
$$


On the other hand,

$$
\langle B D\rangle=\langle B C\rangle\langle C D\rangle=a b
$$

and

$$
\left\langle B^{\prime} D^{\prime}\right\rangle=\langle B D\rangle^{\sim}=b^{\sim} a^{\sim}
$$

but also

$$
\left\langle B^{\prime} D^{\prime}\right\rangle=\left\langle B^{\prime} C^{\prime}\right\rangle\left\langle C^{\prime} D^{\prime}\right\rangle=a^{\sim}\left\langle C^{\prime} D^{\prime}\right\rangle .
$$

So

$$
b^{\sim} a^{\sim}=a^{\sim} d .
$$

Hence, there exists a primitive word (which is necessarily $\alpha$ ) such that $d \in \alpha^{+}$and a conjugate of $\alpha$ (which is necessarily $\alpha_{c}$ ) such that $b^{\sim} \in \alpha_{c}^{+}$and $a^{\sim} \in \alpha_{c}^{*} \alpha_{2}=\alpha_{2} \alpha^{*}$ (here we use the fact that $a$ and $c$ are not both empty). So, $\left\langle B A^{\prime}\right\rangle=\langle B C\rangle\left\langle C A^{\prime}\right\rangle$ and

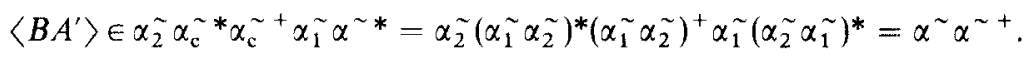

In the same way we have $\left\langle B^{\prime} A\right\rangle \in \alpha_{c} \alpha_{c}^{+}$.

So the result is proved (changing $\alpha$ in $\alpha_{c}$ ) and can be explained by the following scheme obtained by an "unfolding" of the contour of $p$ :

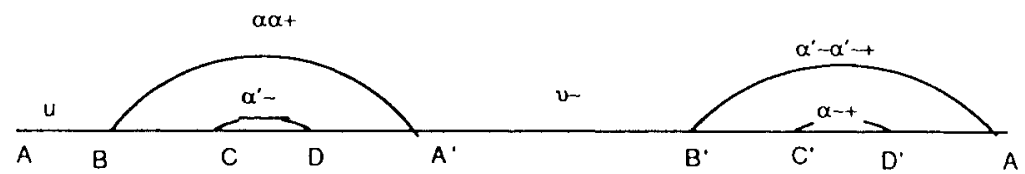

Lemma 4.2. If $\left(A, B, B^{\prime}\right)$ is an exact contact, then we have

$$
\langle A B\rangle=\left\langle A^{\prime} B^{\prime}\right\rangle^{\sim} \quad \text { and }\left\langle B A^{\prime}\right\rangle=\left\langle B^{\prime} A\right\rangle^{\sim}
$$

Proof. It is a clear consequence of Corollary 3.1.

A polyomino $p$ is a pseudoparallelogram $A B A^{\prime} B^{\prime}$ iff there are two points $A$ and $B$ on the boundary of $p$ such that $B \in\left[A A^{\prime}\right]$ and one of the two following conditions is satisfied:

- $\langle A B\rangle=\left\langle A^{\prime} B^{\prime}\right\rangle^{\sim}$ and $\left\langle B A^{\prime}\right\rangle=\left\langle B^{\prime} A\right\rangle^{\sim}$ or

- $\langle A B\rangle=\left\langle A^{\prime} B^{\prime}\right\rangle^{\sim}$, and there is a prime word $\alpha$ and a conjugate word of $\alpha$, $\bar{\alpha}$ such that $\left\langle B A^{\prime}\right\rangle \in \alpha \alpha^{+}$and $\left\langle B^{\prime} A\right\rangle \in \bar{\alpha}^{\sim} \bar{\alpha}^{{ }^{+}}$(we can observe that a pseudoparallelogram is a particular exact polyomino). 
So Lemmas 4.1 and 4.2 have a corollary:

Corollary 4.1. If $\left(A, C, B^{\prime}\right)$ and $\left(A, D, B^{\prime}\right)$ are two different contacts, then $p$ is a pseudoparallelogram $A B A^{\prime} B^{\prime}$.

We now formulate three technical lemmas about pseudoparallelograms which we shall need later.

Lemma 4.3. Let $A B A^{\prime} B^{\prime}$ be a pseudoparallelogram $p$ and let $\mathbf{P}$ be a tiling of the plane by $p$. Let $r, q, s$ be three polyominoes in the tiling, with p type, such that:

- $[r, q]=\left[C A^{\prime}\right], C \in\left[B A^{\prime}\right]$,

- $[q, s]=\left[D A^{\prime}\right], D \in\left[B A^{\prime}\right], D \neq B$,

- $C \neq D$ and $\left|\left\langle B^{\prime} C^{\prime}\right\rangle\right| \geq|\alpha|$ where $\alpha$ is the prime word such that $\left\langle B A^{\prime}\right\rangle \in \alpha \alpha^{+}$.

Under these hypothesis the polyomino $r_{A B^{\prime}}$ belongs to the tiling.

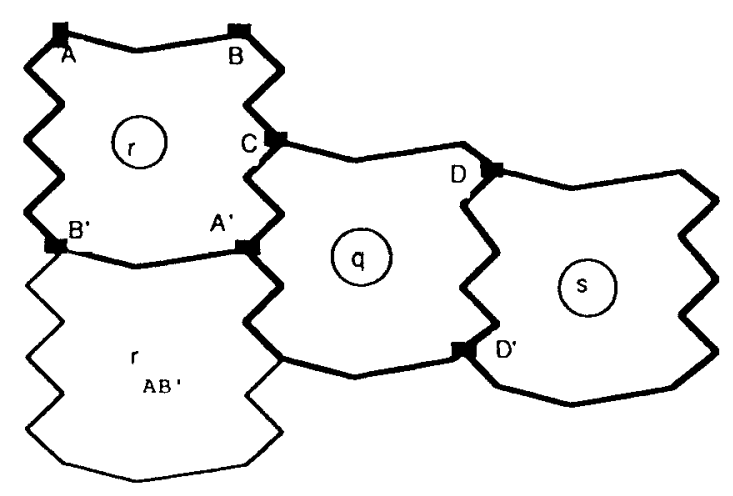

Lemma 4.4. Let $A B A^{\prime} B^{\prime}$ be a pseudoparallelogram $p$ and let $\mathbf{P}$ be a tiling of the plane by $p$. Let $q$ and $r$ be two polyominoes in the tiling such that:

- $[r, q]=\left[C A^{\prime}\right], C \in\left[B A^{\prime}\right]$,

- $|\langle B C\rangle|$ is more than or equal to the maximum of the length of a boundary between any two adjacent polyominoes in this tiling.

Then $r_{A B^{\prime}}$ belongs to the tiling.

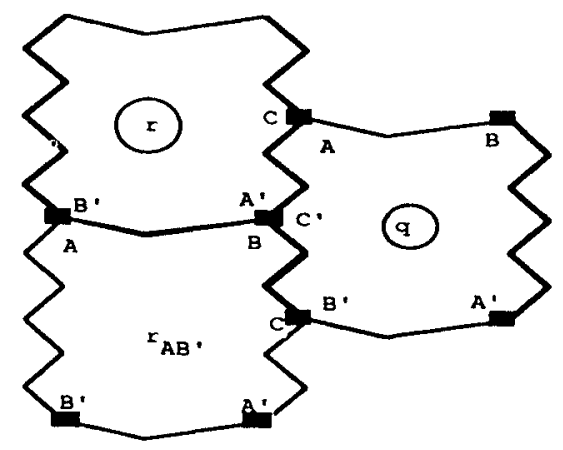


Lemma 4.5. Let $A B A^{\prime} B^{\prime}$ be a pseudoparallelogram $p$ and let $\mathbf{P}$ be a tiling of the plane by $p$. Let $r, q$, s be three polyominoes in this tiling such that:

- $[r, q]=[B C], C \in\left[B A^{\prime}\right], C \neq A^{\prime}, C \neq B$,

- $[q, s]=\left[D A^{\prime}\right], D \in\left[B A^{\prime}\right], C \neq D$.

Then the polyomino $q_{A B}$, belongs to the tiling.

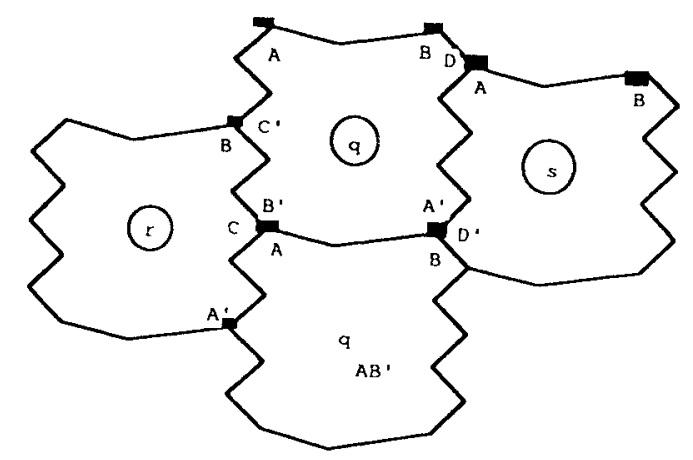

Now we are able to give the last main statement of this paper, and the steps of the proof.

Theorem 4.1. If $p$ is an exact polyomino, every tiling of the plane by $p$ is half-periodic.

\section{Sketch of the proof.}

Step 1. The first step is the proof of a main lemma on which the remainder of the proof depends.

Lemma 4.6. Let $\mathbf{P}$ be a tiling of the plane by $p$. Let $q$ be a polyomino of this tiling and let $q_{u_{1}}, q_{u_{2}}, q_{u_{3}}, q_{u_{4}}$ be a sequence of four adjacent polyominoes of the surrounding of $q$ in $\mathbf{P}$ such that $\left[q_{u_{2}}, q_{u_{3}}\right]$ is of maximal length in the set of boundaries of two adjacent polyominoes in $\mathbf{P}$. Then the following properties are satisfied:

(a) $u_{1}=u_{2}-u_{3}$ or $u_{4}=u_{3}-u_{2}$.

(b) If one of the two equalities does not hold, then $p$ is a pseudoparallelogram.

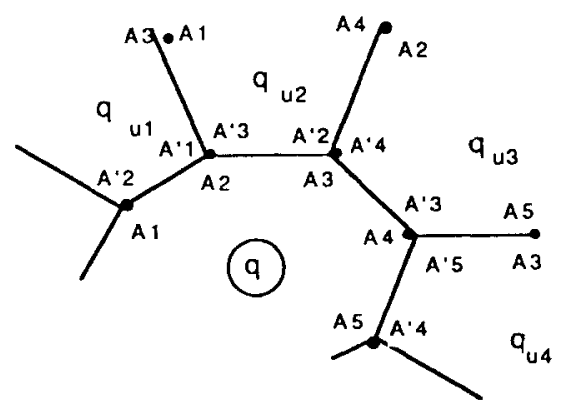




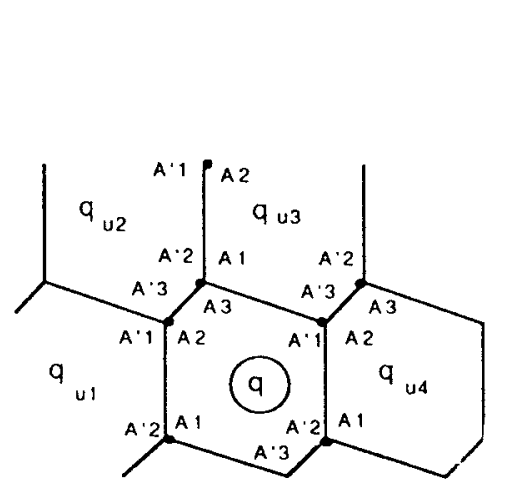

Fig. 1.

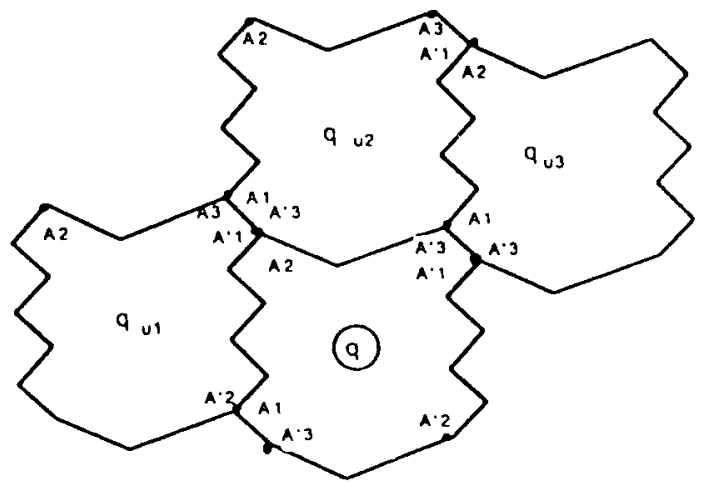

Fig. 2.

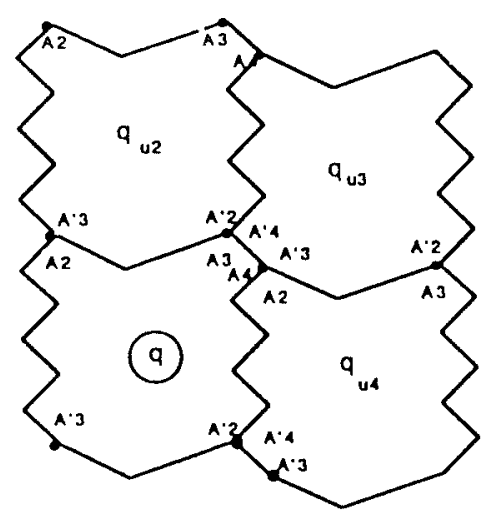

Fig. 3.

The proof of this lemma uses the results of Lemmas 3.1, 3.2, 4.1, and 4.2. We suppose that $u_{1} \neq u_{2}-u_{3}$ and $u_{4} \neq u_{3}-u_{2}$ and we prove that it implies that the boundary of $p$ is not a simple closed curve.

Definition. If $u_{1}=u_{2}-u_{3}$ and $u_{4}=u_{3}-u_{2}$, the boundary vector $u_{3}-u_{2}$ is said to be propagated on both sides from the pair $\left(q_{u_{2}}, q_{u_{3}}\right)$ onto $q$ (Fig. 1). If $u_{1}=u_{2}-u_{3}$ and $u_{4} \neq u_{3}-u_{2}$, the boundary vector $u_{3}-u_{2}$ is said to be propagated on one side (on the left or on the right) onto $q$ (Figs. 2 and 3 ).

Step 2. At this step, two cases have to be considered:

Case 1 . Every time the maximal boundary vector $u_{3}-u_{2}$ appears in the tiling $\mathbf{P}$, it is propagated on both sides onto the two polyominoes at each end of the boundary.

Case 2. There exist at least two polyominoes $r$ and $s$ in the tiling such that $s=r_{u_{3}-u_{2}}$ and the boundary vector $u_{3}-u_{2}$ is propagated on one side from the pair $(r, s)$ at one end of the boundary.

Case 1. Using Lemmas 3.1 and 4.1 it is proved that, for each polyomino $t$ in the tiling, $t_{u_{4}}$ belongs to the tiling and so $\mathbf{P}$ is half-periodic. 


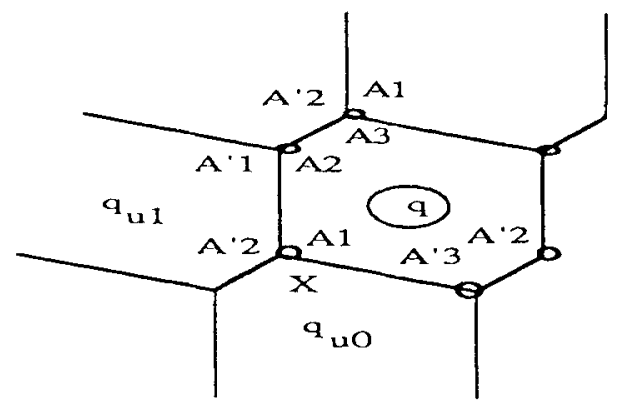

Let $\left(A_{2}^{\prime}, A_{1}, X\right)$ be the contact of the triad $\left(q_{u_{1}}, q, q_{u_{0}}\right)\left(q_{u_{0}}\right.$ is the polyomino adjacent to $q_{u_{1}}$ in the surrounding of $q$, before $q_{u_{1}}$ ). We prove that:

If $\left\langle A_{2}^{\prime} A_{1}\right\rangle$ is a prime word, then $x=A_{3}^{\prime}$ and,

in that case, the tiling is regular (Fig. 4).

If $X \neq A_{3}^{\prime}$, then $\left\langle A_{2}^{\prime} A_{1}^{\prime}\right\rangle$ is not primitive and

the tiling is $u_{4}$ invariant (Fig. 5).

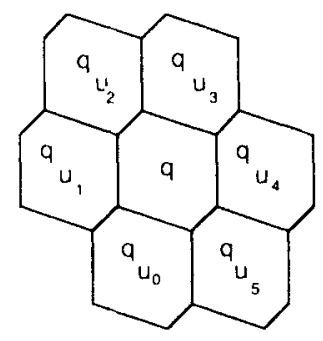

Fig. 4.

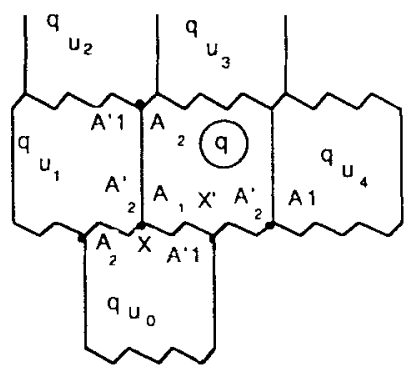

Fig. 5.

Case 2. We can suppose that $u_{1} \neq u_{2}-u_{3}$ and $u_{4}=u_{2}-u_{3}$ (Fig. 2). Using Lemmas 4.3 and 4.4 we deduce that $q_{u_{1}-u_{2}}$ belongs to $\mathbf{P}$.

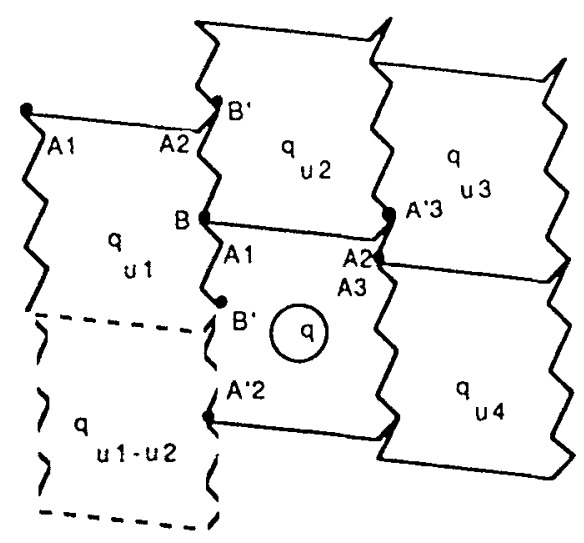




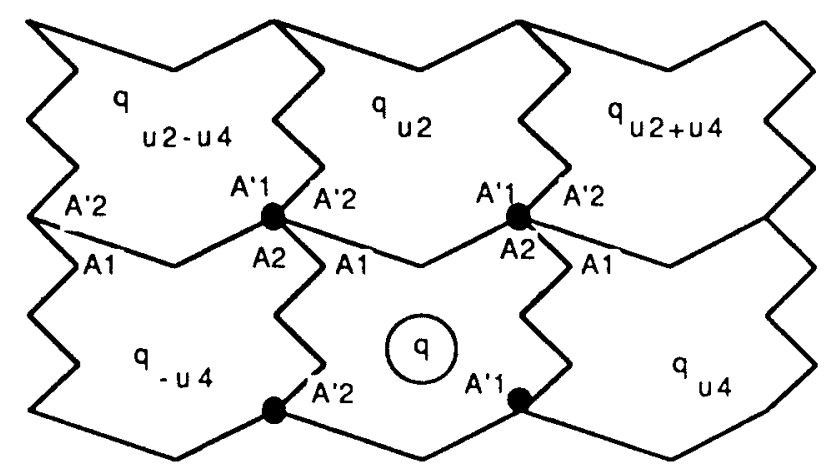

Fig. 6.

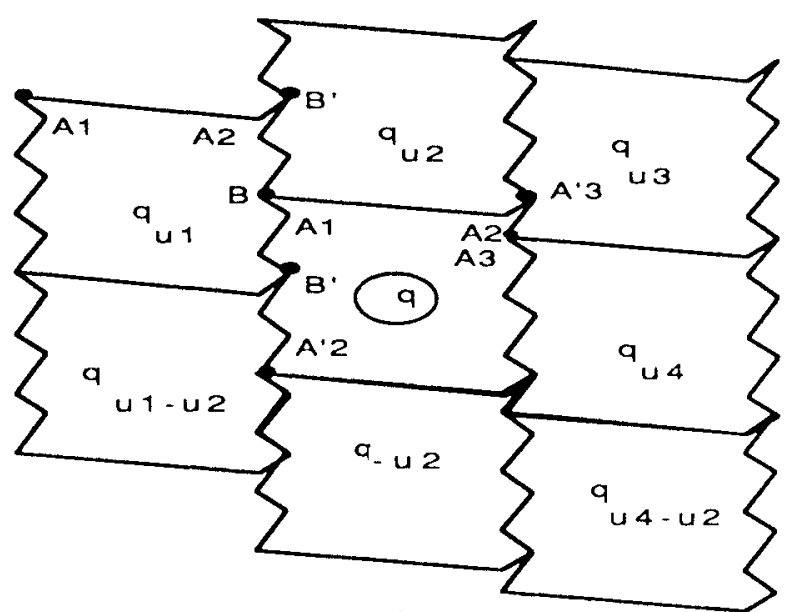

Fig. 7 .

Let $\left(B, A_{2}^{\prime}, A_{1}\right)$ be the contact of the triad $\left(q_{u_{1}}, q_{u_{2}}, q\right)$. Then we prove (using previous lemmas) that:

If $B=A_{1}^{\prime}$, then the tiling is $u_{2}$ or $u_{4}$ or both $u_{2}$ and $u_{4}$ invariant (Fig. 6). (III)

$$
\text { If } B \neq A_{1}^{\prime} \text {, then the tiling is } u_{2} \text { invariant (Fig. 7). }
$$

So the proof is achieved.

\section{Surroundings of an Exact Polyomino}

The different lemmas established in the previous section are now very useful to describe all the complete surroundings of an exact polyomino.

Theorem 5.1. Every complete surrounding of an exact polyomino contains six, seven, or eight polyominoes, and the minimal surrounding extracted from the complete one contains, respectively, six, five, or four polyominoes. 
Proof. First, by Corollary 3.1 every surrounding can be extended in a tiling of the whole plane, so we have only to look at the surroundings appearing in the tilings of the plane. However, in Theorem 4.1 we proved that every tiling is of one of the types described in (I) (IV). In cases (I) and (II), each complete surrounding contains six polyominoes and is minimal. In cases (III) and (IV) every complete (minimal) surrounding has six, seven, or eight (resp. six, five, or four) polyominoes.

We give below the different complete and, respectively, minimal surroundings of an exact polyomino.

Complete 6-surroundings (they are also minimal).

- $\left\langle B A^{\prime}\right\rangle=\left\langle B^{\prime} A\right\rangle^{\sim}$.

- There exists a word $\alpha=\alpha_{1} \alpha_{2}$ such that:

$$
\begin{array}{ll}
\langle A C\rangle \in \alpha^{*} \alpha_{1}, & \langle C B\rangle \in \alpha_{2} \alpha^{*}, \\
\left\langle A^{\prime} D\right\rangle \in \alpha_{1}^{\tilde{\alpha}} \alpha^{\sim *}, & \left\langle D B^{\prime}\right\rangle \in \alpha^{\sim *} \alpha_{2}^{\tilde{}} .
\end{array}
$$

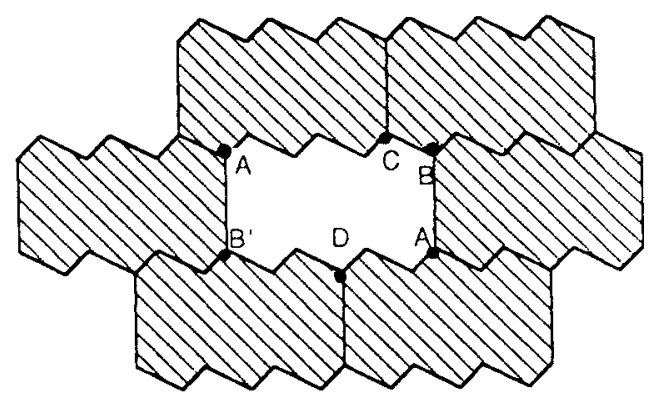

Complete 7-surroundings (the associated minimal ones contain five polyominoes).

- $\left\langle B A^{\prime}\right\rangle=\left\langle B^{\prime} A\right\rangle^{\sim}$.

- There exists a word $\alpha$ such that:

$$
\langle A B\rangle \in \alpha \alpha^{+}, \quad\left\langle A^{\prime} C\right\rangle \in \alpha^{\sim+}, \quad\left\langle C B^{\prime}\right\rangle \in \alpha^{\alpha^{+}} \text {. }
$$

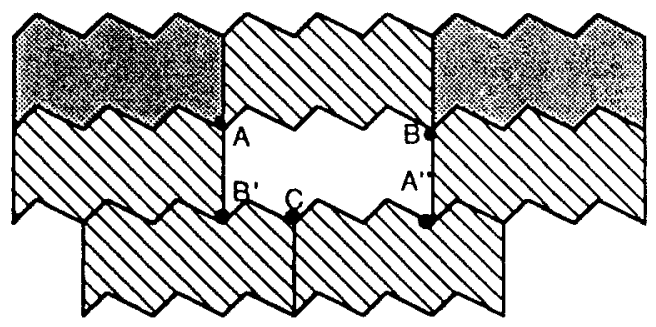


Complete 8-surroundings (the associated minimal ones contain four polyominoes).

- $\langle A B\rangle=\left\langle A^{\prime} B^{\prime}\right\rangle^{\sim},\left\langle B A^{\prime}\right\rangle=\left\langle B^{\prime} A\right\rangle^{\sim}$.

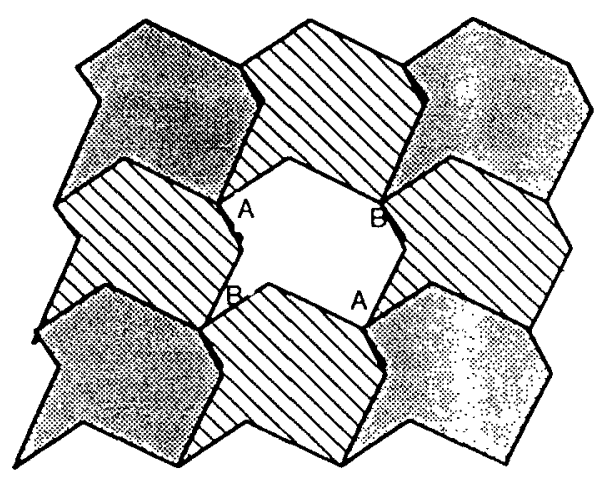

From this description we immediately deduce the following property:

Proposition 5.1. If a polyomino has a complete surrounding with seven polyominoes, then it also has a complete surrounding with six or eight polyominoes.

\section{References}

1. S. W. Golomb, Polyominoes, Allen and Unwin, London (1966).

2. B. Grünbaum and G. C. Shephard, Tilings and Patterns, Freeman, New York (1986).

3. R. Penrose, Pentaplexity, Eureka 39 (1978).

4. R. M. Robinson, Undecidability and nonperiodicity of tilings of the plane, Invent. Math. 12, 177-909 (1971).

5. H. D. Shapiro, Theoretical limitations on the efficient use of parallel memories, IEEE Trans. Comput. (1978).

6. H. Wang, Games, logic and computers, Scientific American (1965).

7. H. A. G. Wijshoff and J. Van Leeuven, Arbitrary versus periodic storage schemes and tessellations of the plane using one type of polyomino, Inform. Control 62, 1-25 (1984).

Received June 15, 1989, and in revised form February 20, 1990, and February 15, 1991. 